\title{
The Disign of CMOS Integrated Acceleration Detection Circuit System
}

\author{
Yijiang Cao, Jiamu Cao, Zhefei Wang and Shan Li \\ Electronic Science and Technology, College of applied science, Harbin University \\ of science and technology, Harbin, China \\ caoyijiang@hrbust.edu.cn, leocaojiamu@126.com,wzff8909@163.com
}

\begin{abstract}
In this dissertation, we designed a kind of CMOS integrated precision acceleration detection system which is use high performance operation amplifier as the core. It was analysis directly as a low noise accelerometer and the principle of charge amplification. Detection circuit is based on the principle of time detection, which can be detected variation of the acceleration continuously in a successive time, and improving the detection accuracy. The input terminal of the circuit using sensitive charge amplifier to realize $C-V$ conversion, which is effectively avoid the switch noise of detection principle. The whole circuit is designed and simulated based on $0.5 \mu \mathrm{m}$ CMOS process. The research result of Operational Amplifier module has shown that, the circuit's the DC open-loop gain is $85.96 \mathrm{~dB}$, and its phase margin is $62.8^{\circ}$, and the effect input noise is $3.632 \mathrm{nV} / \sqrt{\mathrm{Hz}}$. The research result of overall current has shown that, full scale range of the closed-loop system between $\pm 2.5 \mathrm{~V}$ the voltage is $\pm 30 \mathrm{~V}$. The detection sensitivity is $16.2 \mathrm{mV} / \mathrm{g}$, which is enough to conform the requirements of the low noise accelerometer.
\end{abstract}

Keywords: acceleration; detection; low noise; charge amplifier

\section{Introduction}

MEMS micro accelerometer is a kind of important inertial device which is mainly used for sensing the moving vehicles along a certain direction' s motion information, in order to get the data of acceleration, speed, displacement and so on. By virtue of the advantages, such as small volume, light weight, low consumption, low cost and easy integration, it has become the core of the micro inertial measurement element, and it has been widely used in the automotive, consumer electronics, aerospace, seismic exploration and other fields [1]. For the detecting of the external acceleration, we need to match the detection circuit, and modulate the changes in capacitance caused by changes external acceleration to voltage change. However, the volume of the acceleration sensor is always very small, leading to its small capacitance, when the systems can effected by external, the capacitance changes of itself will be very small [2]. Therefore, in order to ensure the stability of the system performance, it is necessary to design a detection circuit with high performance parameters. Charge amplifier as the access module of MEMS capacitive accelerometer, it changed the weak mechanical output capacitance into voltage signal. The noise introduced by the charge amplifier will be enlarged by the subsequent circuit, so low-noise charge amplifier is necessary for high sensitivity acceleration detection system circuit [3]. 


\section{Principles}

\subsection{Testing Principles of Accelerometer}

As shown in Figure 1 the structure of common micro accelerometer is generally made up of sensitive quality block, cantilever beam, shell (there are upper and lower electrode plate) and the detection circuit and other parts. Discharge electrodes takes the glass with a certain metal film as a conducting plate and is exported by a special export lead, the middle sensing element is made up of monocrystalline silicon material, because of the spring characteristic of cantilever beam, middle quality piece is movable , the fixed discharge electrodes and middle moving electrode is wrapped in together by ionic bond process which constitute two up and down differential condenser. When the object under test by external acceleration, sensing element will lead to relative motion between the block and shell, the change of the space between sensitive element and the testing electrodes changes will cause the change of equivalent capacitance [4].

$$
g_{m}=\sqrt{2 \mu C_{o x} \frac{W}{L} I_{d s}}
$$

By the formula (1), we can see that the size and direction of relative displacement has a corresponding relation with the size and direction of acceleration [5], we can get the size and direction of acceleration by means of measuring the change of differential capacitance, $I_{d s}$ ( $\Delta C$ ) is the micro variable capacitor, $\mathrm{C}$ is the capacitance between plates, $d_{0}$ is the distance between the plate and the sensitive element, $m_{\text {is }}$ the quality of sensitive element, $K_{z}$ is the equivalent stiffness of the beam.

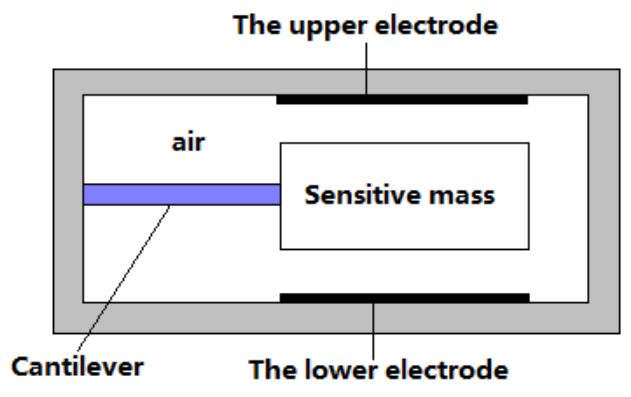

Figure 1. Structure Diagram of Micro Accelerometer

\subsection{Principles of Charge Amplifier}

Charge amplifier can convert the change of differential capacitance which is outputted by mechanical structure to voltage signal output, and at the same time import voltage feedback signal to realize the working of closed-loop system [6]. Operational amplifier constitutes a form similar to the integrator through the feedback capacitance and feedback resistance, when mechanical structure differential capacitance has a differential change there will be a charge flowing to the feedback capacitance, charge amplifier coverts differential capacitance to variation of voltage by integrating of charge [7]. Figure 2 is the circuit's principle diagram of the charge amplifier. Charge amplifier consists of an operational amplifier, a feedback capacitance $C_{f}$ and a feedback resistance $R_{f}$, and $V_{i n-}, V_{i n+}$ is a carrier signal having the same amplitude and 180 difference of phase. ${ }^{\text {in }}$ is 
the midpoint voltage of differential capacitance, $V_{T}$ is the bias voltage of the amplifier at the same input port, in ideal conditions, $V_{\text {in }}$ virtual geomagnetic. When there is no acceleration $(\Delta C=0), V_{\text {out }}=V_{T}$. When the changes of acceleration $(\Delta C \neq 0)$ is detected, we have

$$
V_{\text {out }}=-\frac{2 \Delta C}{C_{f}} U \sin \omega t+V_{T}
$$

Angular frequency $\omega>1 / R_{f} C_{f}$, thus

$$
\Delta V_{\text {out }}=-\frac{2 \Delta C}{C_{f}} U \sin \omega t
$$

The sensitivity of the charge amplifier circuit:

$$
\left|\frac{\Delta V_{\text {out }}}{\Delta C}\right|=\frac{2 U}{C_{f}}
$$

We can see from formulary (4) that the smaller the capacitance value is, the greater the gain will be, but at the same time, it will cause the input signal more vulnerable to feedback signal, lower the noise characteristic of system. Also there is a compromise on the feedback resistance, if you want to reduce the noise introduced by the charge amplifier, and this demands of increasing the feedback resistance value [8]. If considering the feedback signal on the output side as far as possible to attenuate, the feedback resistance value demands to be reduced.

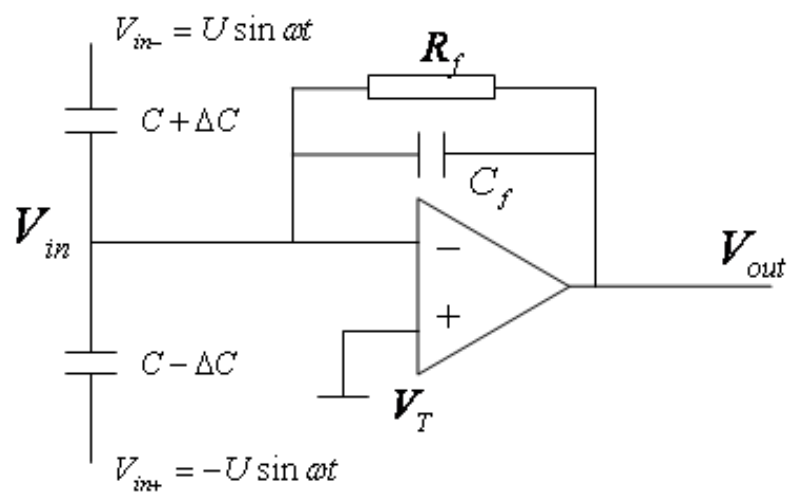

Figure 2. Principle Diagram of Charge Amplifier Circuit

\section{Detection System}

The requirement of closed-loop accelerometer noise characteristics is often higher. This design uses sensitive charge amplifier feedback system to make sensor feedback work. The overall Schematic of charge amplifier feedback Schematic was shown as Figure 3.

When input acceleration signal modulated by the mechanical structure, slight capacitance differential is converted into a voltage change, because the differential capacitance of the mechanical part is relatively small, cause the voltage change of conversion is small [9], in order to facilitate subsequent circuit detection and easy to read the simulation data, use the proportional amplifier non-inverting after sensitive charge amplifier, then demodulated output signal, modulation the signal into a mixed-signal 
which contains low frequency components and high frequency components, the highfrequency part of the signal is filtered by low-pass filter, the low frequency signal which is proportional to the acceleration will be kept. After regulated by PI proportional integrator, the output signals feedback to the non-inverting terminal of the sensitive charge amplifier. Because the principle of virtual circuit, The potential of the movable electrode which is connected to sensitive charge amplifier will be changed with the feedback voltage, in order to achieve the feedback of the signal.

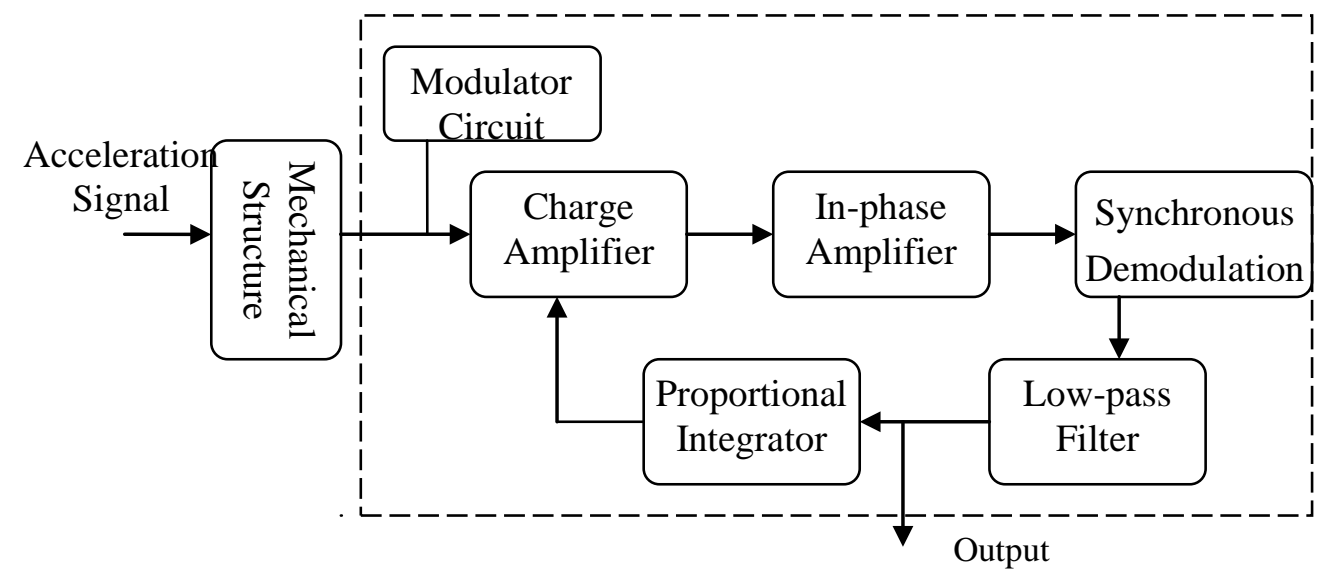

Figure 3.The Overall Scheme Diagram of Detection Circuit

\section{Design of Charge Amplifier Circuit}

Noise which is introduced by the first stage at the charge amplifier is amplified by the driver stages and final stages, noise introduced by the second stage is only amplified by the final stages and it is negligible if compared with the noise introduced by first stage, so the first stage of charge amplifier is particularly important. Figure 4 is the closed loop system injected by multistage noise, among them, $A_{1}, A_{2}$ is the gain of first and second stage respectively. $V_{n 1}, V_{n 2}$ represent the input noise of first and second stage. $\beta$ is the feedback factor.

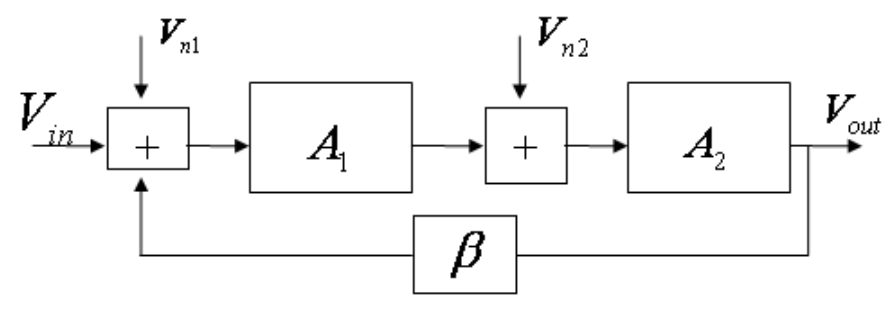

Figure4. Analysis of Closed-loop System Noise

$$
V_{\text {out }}=\frac{V_{\text {in }}+V_{n 1}+V_{n 2} / A_{1}}{1 / A_{1} A_{2}+\beta}
$$

We can see from formular (5), If $A_{1}$ is large enough, $V_{n 2}$ can be can be negligible. The noise sources of MOS tube thermal noise is generated in the channel, for the channel noise of long channel MOS devices which work in a saturated region channel, we can use the current source which is connected on a connection on both ends of drain-source to simulate, and its spectral density is 


$$
I_{n}^{2}=4 k T \gamma g_{m}
$$

The coefficient of long channel transistor $\gamma=2 / 3, g_{m}$ is the tran conductance input transistors, flicker noise is simulated by the voltage source connected in series to gate:

$$
V_{n}^{2}=\frac{K}{C_{o x} W L} \frac{1}{f}
$$

$\mathrm{K}$ is a constant related to process, its order of magnitude is $10^{-25} \mathrm{~V}^{2} \mathrm{~F}$. From the formular (7) we find that the noise spectral density is inversely proportional to the frequency, so the flicker noise is also called noise [10]. The output noise voltage:

$$
V_{n, \text { out }}^{2}=\left(4 k T \frac{2}{3} g_{m}+\frac{K}{C_{o x} W L} \frac{1}{f} g_{m}^{2}\right) R_{D}^{2}
$$

The equivalent input noise voltage $V_{n, i n}$ :

$$
\begin{gathered}
V_{n, \text { in }}^{2}=\frac{V_{n, \text { out }}^{2}}{A_{v}^{2}} \\
=\left(4 k T \frac{2}{3} g_{m}+\frac{K}{C_{o x} W L} \frac{1}{f} g_{m}^{2}\right) R_{D}^{2} \frac{1}{g_{m}^{2} R_{D}^{2}}=\frac{8}{3} k T \frac{1}{g_{m}}+\frac{K}{C_{o x} W L} \frac{1}{f}
\end{gathered}
$$

With the increase of frequency, channel thermal noise account for most of the noise of high frequency. In order to reduce the thermal noise, the tran conductance of the input transistor must be increased,

$$
g_{m}=\sqrt{2 \mu C_{o x} \frac{W}{L} I_{d s}}
$$

As seen from formular (10), the tran conductance can be changed by changing the ratio of its width to length or bias current. Due to the limit of CMOS technology, L can not be less than the minimum channel length, only increasing the channel width so as to increase input capacitance, or increasing the bias current $I_{d s}$ to increase $g_{m}$ [11].

Figure 5 is the operational amplifier circuit in charge amplifier, the first stage of the operational amplifier PMOS differential input folding cascade amplification circuit composed of M1 M10 and M12 M17. Taking the constant current and a current mirror as a source of current, M1 is in-phase input, M2 is inverting input. M11, M18 constitute the second amplifying circuit. Cascade structure has a high output impedance, not only can provide high gain, but also has a "block" effect, and make the input device not susceptible to the changing of the output node voltage[12]. This structure is usually divided into sleeve and folding. Sleeve has a series advantages such as high gain, low power consumption, good frequency characteristic. But the output swing is low, folding is characterized by a high output swing, common-mode input range, etc. But the gain is relatively low. In order to have a high output swing and a larger common model range, folding cascade structure is used, its relatively low gain problem can be solved by two stages of op-amp structure.

In this design, in order to reduce the noise of the op-amp, we selects the PMOS transistor as a differential input tube, because of the noise of the PMOS transistor is about 2-5 times less than NMOS. We choose greater $\mathrm{L}$; increase the WL area to reduce the equivalent input noise at the time of design input PMOS tube [13]. To make the grid 
length of load transistor greater than the length of input transistor so as to minimize noise. Selecting a smaller value as the Overdrive voltage of input tube M1, M2 which is $0.2 \mathrm{~V}$, so that in the same bias current we can obtain a high Tran conductance $g_{m}$. Load tube M12, M13 select greater values by virtue of driving voltage such as $0.4 \mathrm{~V}$, to reduce the influence of load tube to input equivalent noise. As for the MOS tube which provide current source of bias current at various stages and constitute current mirror, we design it into a small breadth length ratio and a greater driving voltage to reduce the influence of the equivalent input noise and meet the requirements of devices working in saturated zone [14].

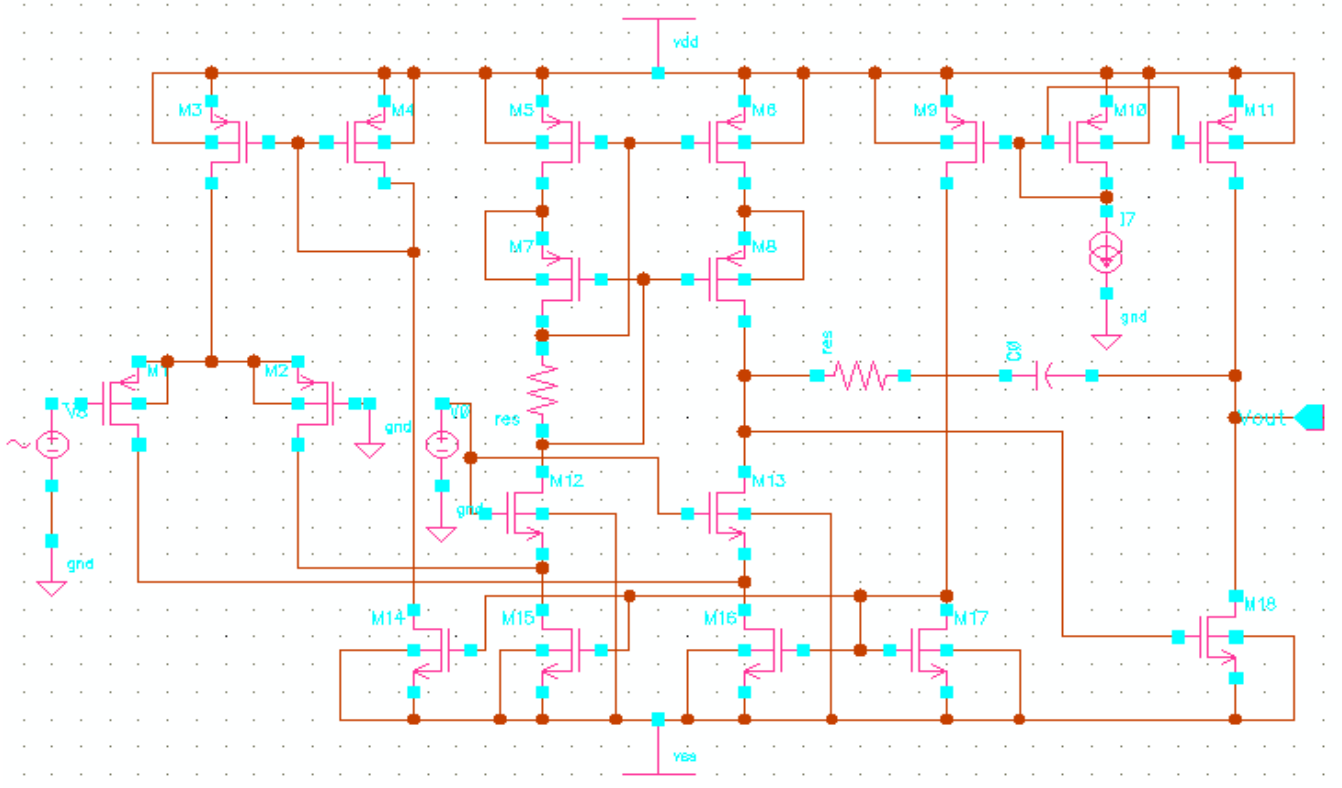

Figure 5. Diagram of the Operational Amplifier Circuit in Charge Amplifier

Through the analysis and calculation, we set the size and relevant parameters of MOS tube based on $0.5 \mu \mathrm{m}$ CMOS technology library. Using simulation software to simulate the whole circuit for times and make a corresponding adjustment to the breadth length ratio of each MOS tube, after adjusting and optimizing, the size of each MOS tube is shown in Table 1.

Table 1. MOS Transistor Parameters

\begin{tabular}{|l|l|l|l|}
\hline \multirow{4}{*}{ Transistor Type } & \multicolumn{3}{|c|}{ Transistor Parameters } \\
\cline { 2 - 4 } & \multicolumn{1}{|c|}{ Number } & Multiplier & \multicolumn{1}{|c|}{ W/L } \\
\hline PMOS tube & M1、M2 & 60 & $30 / 2$ \\
\cline { 2 - 4 } & M3、M4 & 10 & $40 / 4$ \\
\cline { 2 - 4 } & M5、M6、M7、M8 & 40 & $5 / 2$ \\
& M9、M10 & 5 & $8 / 4$ \\
& M11 & 70 & $20 / 4$ \\
\hline NMOS tube & M12、M13 & 30 & $9 / 3$ \\
\cline { 2 - 4 } & M14 & 70 & $8 / 2$ \\
\cline { 2 - 4 } & M15、M16 & 80 & $16 / 4$ \\
\cline { 2 - 4 } & M17 & 120 & $12 / 4$ \\
\cline { 2 - 4 } & M18 & 4 \\
\hline
\end{tabular}


Figure 6 is amplifier's open loop frequency characteristic, it is observed that operation dc gain of open-loop is $85.96 \mathrm{~dB}$, the unit gain bandwidth is $12.58 \mathrm{MHz}$, phase margin is $62.8^{\circ}$, High magnification can meet demand that there is a micro change of capacitance along with the accelerometer, with the corresponding, there will be a voltage change so as to observation. Figure 7 is the equivalent Input noise characteristic curve of operational amplifier, it can be seen that noise is controlled at the ideal level within the scope of the low frequency, noise $1 / f$ characteristic is mainly embodied in current noise, noise is $3.632 \mathrm{nV} / \sqrt{\mathrm{Hz}}$ within the scope of the low frequency.

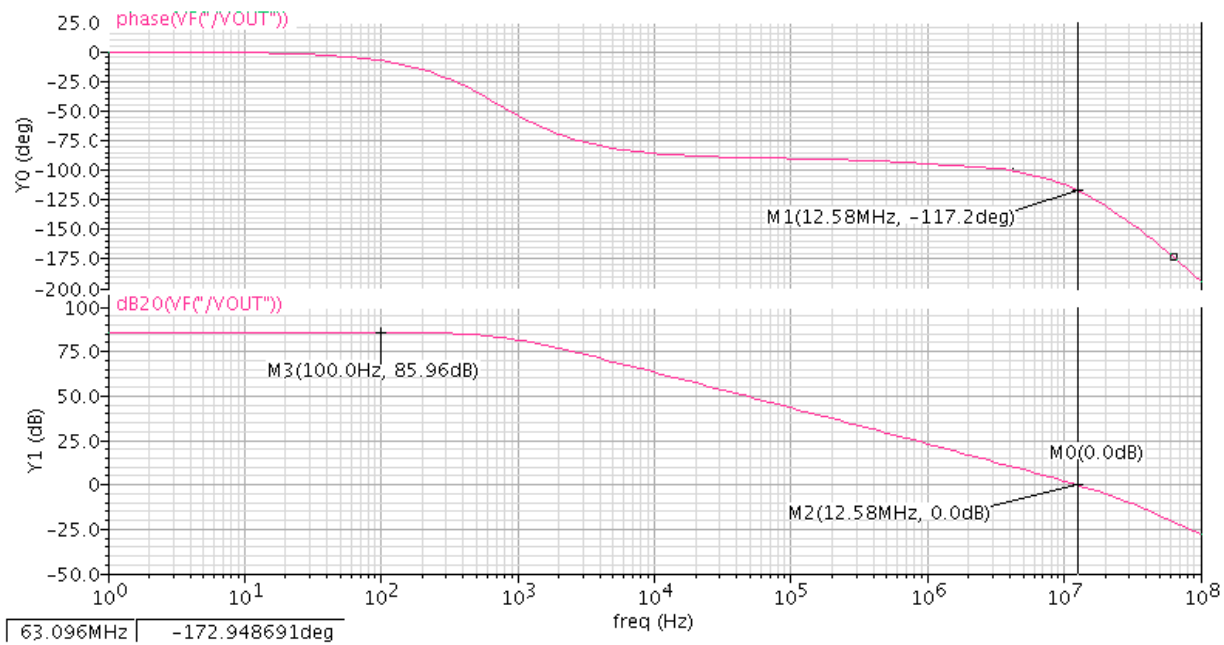

Figure 6. Operational Amplifier's Open Loop Frequency Characteristic

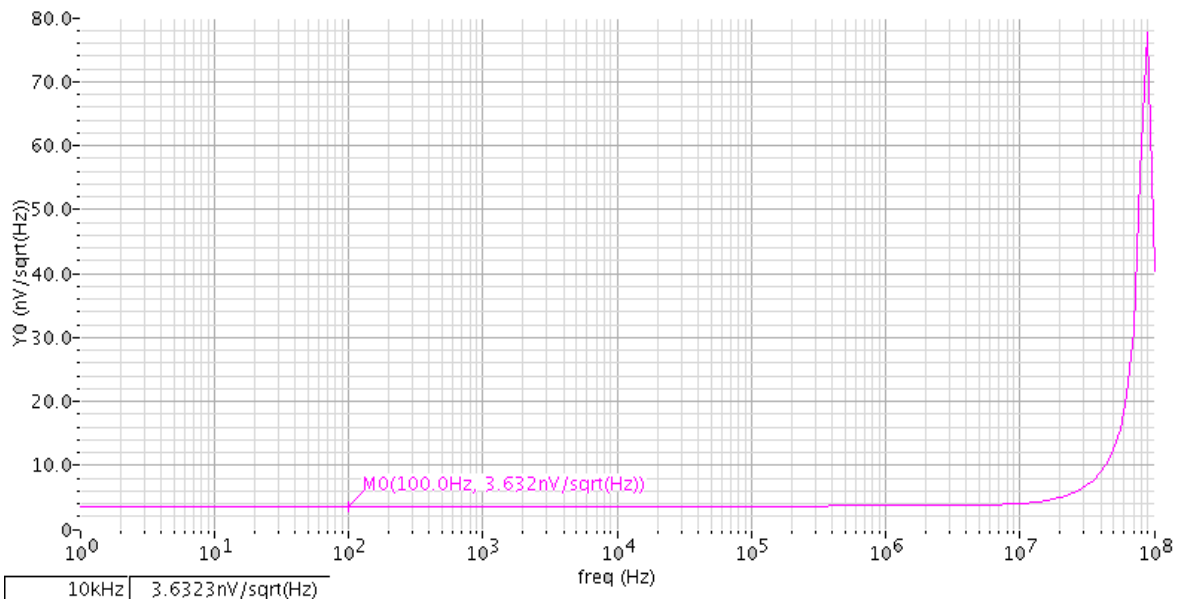

Figure7. Input Equivalent Noise of Operational Amplifier

\section{The Detection System's Overall Circuit Design and Simulation}

After detection circuit for each functional module design and simulation validation, the various sub-modules are connected together to form the whole circuit. We carry out Overall simulation by using simulation software, and adjust the width to length ratio of Sub-module circuit MOS transistor for some non-compliance. And then fished the overall simulation, finally determined all the parameters of MOS transistors. Figure 8 is the overall circuits of acceleration detection system. $\pm 2.5 \mathrm{~V}$ power source is used to provide power. When there is external acceleration, that equivalent to a signal in the electrical model of the mechanical structure, then equivalent capacitance generated a differential capacitance $\Delta C$. When the change in capacitance is process by Modulation circuit, 
sensitive charge amplifier, proportional amplifier non-inverting, synchronous demodulation circuit solution, low-pass filter and proportional integrator, the proportional integrator will maintain the voltage value of former input signal. This feature of accumulation and maintaining, make sure the integral regulator can eliminate the static error of control system. Overall circuit self-test function verification simulation when given public pole plate a the DC detection voltage, self-detection voltage will produce a static electricity on cantilever beam, and make the cantilever beam deviate from the original location, that the same as the addition of acceleration signals. Then there is a fixed $\Delta C$ between the plates, the signal though the detection circuit, should output a DC voltage proportional to $\Delta C$. Simulation results is shown in figure9, when $\Delta C=0.5 \mathrm{pF}$, overall circuit output curve is a, the output voltage is $-32 \mathrm{mV}$. When $\Delta C=1 \mathrm{pF}$, overall circuit output curve is $\mathrm{b}$, the output voltage is $-65 \mathrm{mV}$. When $\Delta C=2 \mathrm{pF}$, overall circuit output curve is c, the output voltage is $-130 \mathrm{~m}$. When $\Delta C=4 \mathrm{pF}$, overall circuit output curve is $\mathrm{d}$, the output voltage is $-260 \mathrm{mV}$.

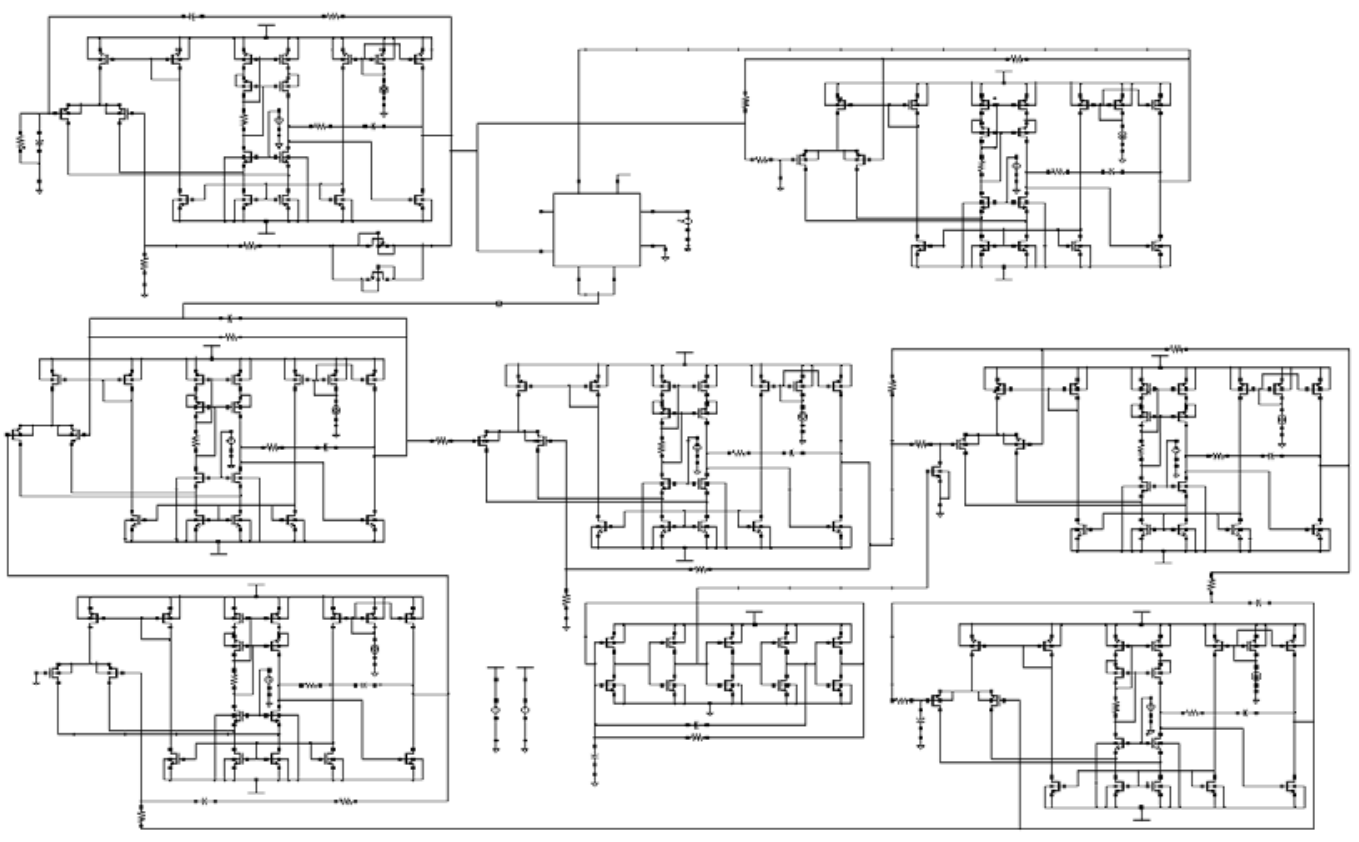

Figure 8.The Overall Circuits Diagram of Acceleration Detection System

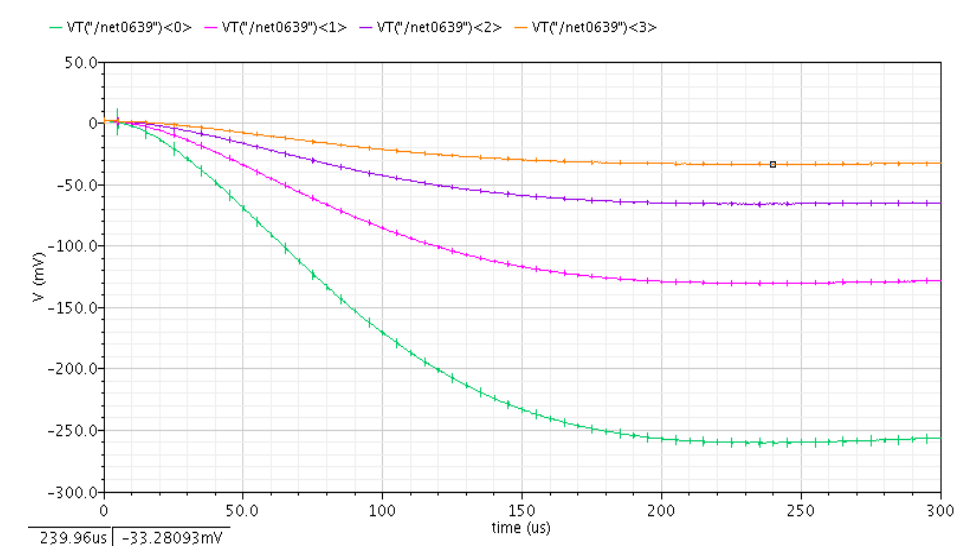

Figure 9.The Results of Transient Simulation when $\Delta C$ were Different 
As shown in Figure 10, when $\Delta C=-0.5 \mathrm{pF}$, the output voltage is $32 \mathrm{mV}$. When $\Delta C=-$ $1 \mathrm{pF}$, the output voltage is $65 \mathrm{mV}$. The simulation results has shown that the relation between output voltage and differential capacitance is linear, the output voltage reflect the change in capacitance effectively, and we can get the polarity of differential capacitance by detect the polarity of voltage value, then we can determined the direction of acceleration.

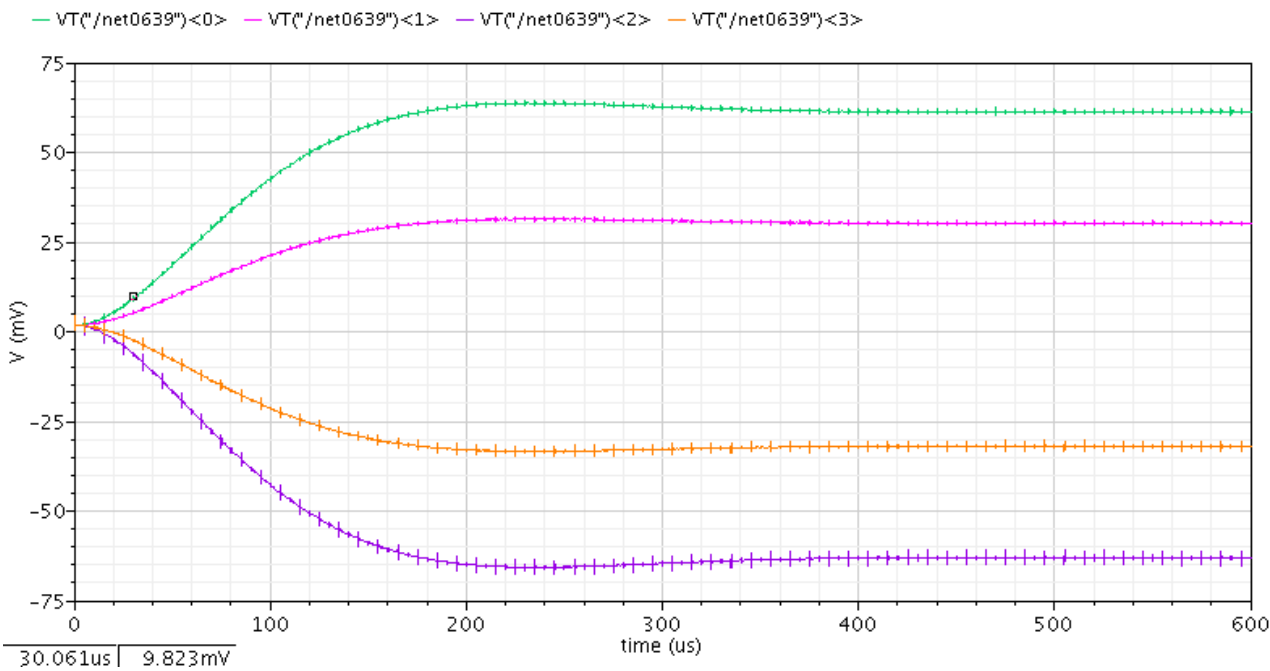

\section{Figure 10.The Results of Transient Simulation when $\Delta C$ take $\pm 0.5 p F, \pm 1 p F$}

As shown in Figure 11, when the mechanical part detected the amplitude is $\pm 10 \mathrm{~g}$, the frequency of sinusoidal acceleration is $3 \mathrm{kHz}$, the signal is modulated to high-frequency signal by modulation circuit, after sensitive charge amplifier, inverting amplifier, synchronous demodulation circuit, and low-pass filter, finally we will get a sinusoidal voltage signal which the margin is $\pm 162 \mathrm{mV}$ and the frequency is $2.5 \mathrm{kHz}$, output voltage is a linear relationship between the acceleration signal. These simulation results shown that the basic function of the acceleration sensor detects circuit has achieved, there are little different between simulation results and theoretical calculations, this design has met the requirements.

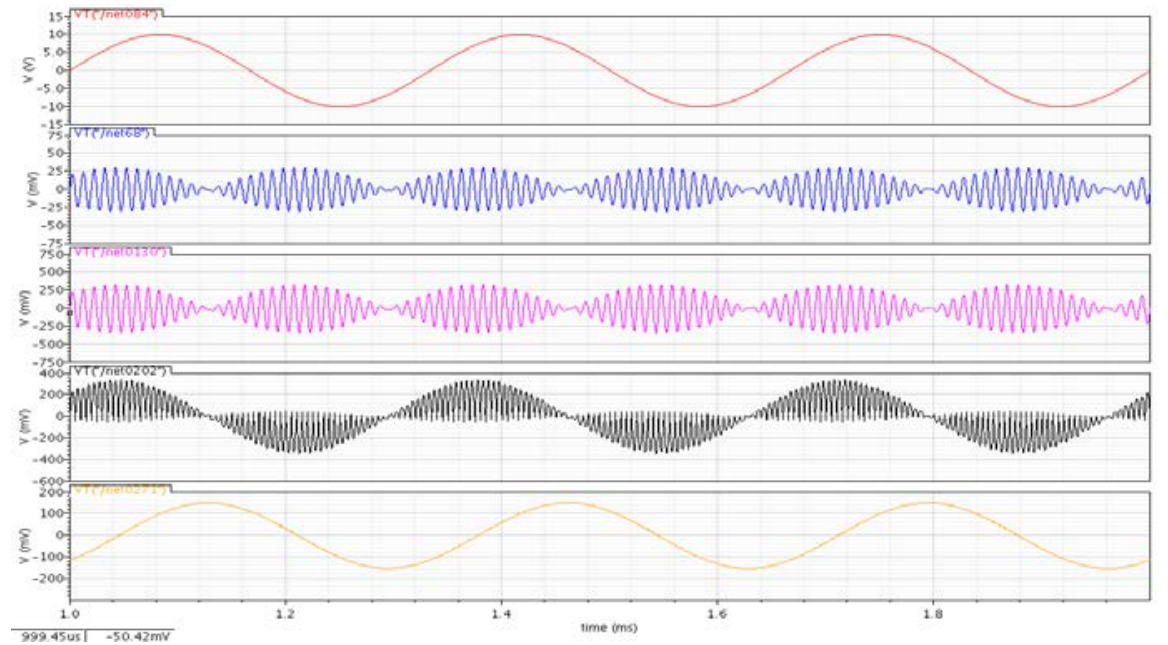

Figure 11.The Results of Whole Simulation When the Outside Acceleration is $\pm 10 \mathrm{~g}$ 


\section{Conclusions}

In this dissertation, we designed a kind of detection system with precision acceleration which is uses low noise, greater gain charge amplifier as the core. Operational amplifier uses PMOS as the amplifier input on the tube, overall adopts two stages operational amplifier of folded cascode, effectively improve the gain of op amp, reducing the equivalent input noise of op amp. C-V Transform .The output voltage signal through the PI proportional integrator, back to the in-phase input end of the charge amplifier and form closed loop, effectively eliminate the static error. The simulations reveal that the full range of the closed loop system is $\pm 30 \mathrm{~g}$ and the detection sensitivity is $16.2 \mathrm{mV} / \mathrm{g}$. Therefore the design meets the requirement of closed-loop acceleration self-test circuit to sensor system, and it can effectively realize the detection of the physical acceleration.

\section{Acknowledgments}

During the period of researching, we greatly thank the Department of Electronic Science and Technology of Harbin University of science and technology and the MEMS Centre of Harbin Institute of Technology for strongly support.

\section{References}

[1] B. Lv, X. Liu, P. Wang and L. Yin, “A Low Noise and Low Temperature Coefficient CMOS Bandgap Reference for MEMS Micro-accelerometer”, Proceedings of the 10th Russian-Chinese Symposium of Laser Physics and Laser Technologies and Academic Symposium on Optoelectronics Technology, Harbin, China, (2010) July 28-August 1.

[2] S. Hong and N. Liu, "Switched capacitor type micro accelerometer capacitance detection circuit design and research”, University of Electronic Science and Technology of China, Chengdu, (2011).

[3] M. Beikahmadi and S. Mirabbasi, "A Low-power Low-noise CMOS Charge-sensitive Amplifier for Capacitive Detectors", Proceedings of the IEEE 9th International of New Circuits and Systems Conference, Bordeaux, France, (2011) June 26-29.

[4] M. Tsai, C. Wang, C. Sun and W. Fang, "A Novel Out-of-Plane Accelerometer with Fully-Differential Sensing Circuit and Sub-Micron Gap”, Proceedings of International Solid-State Sensors, Actuators and Microsystems Conference, Lyon, France, (2007) June 10-14.

[5] K. Lon and C. Luo, "Capacitive Micro-accelerometer PSPICE Simulation Model Research", Proceedings of the International Symposium of Information Science and Engineering, Shanghai, China, (2010) December 24-26.

[6] R. Bassini, S. Brambilla and A. Pullia, "A hybrid low-noise charge sensitive amplifier with fast discharge mechanism”, Proceedings of the IEEE of Nuclear Science Symposium Conference Record, (2001) November 4-10.

[7] M. Kollar and L. Michaeli, "Consideration for a New Micromachined Accelerometer Capacitive Interface Based on Switched Flip-Flop Circuit", Proceedings of the IEEE 9th International of Instrumentation and Measurement Technology Conference, Sorrento, Spain, (2006) April 24-27.

[8] H. Sun, K. Jia, X. Liu and G. Yan, "A Low Temperature-dependence Gain-boosting Front-end Amplifier for CMOS-MEMS Gyroscopes”, Proceedings of the IEEE Sensors Conference, Kona, America, (2010) November 1-4.

[9] C. Condemine, N. Delorme, J. Soen and J. Durupt, “A 0.8ma 50Hz 15b SNDR $\Delta-\sum$ closed-loop 10g Acclerometer Using an 8th-order Digital Compensato", Proceedings of the IEEE International of SolidState Circuits Conference, San Francisco, America, (2005) February 10.

[10] J. Wu, G. K. Fedder and L. R. Carley, “A Low-noise Low-offset Capacitive Sensing Amplifier for a 50$\mu \mathrm{g} / \mathrm{Hz}$ Monolithic CMOS MEMS Accelerometer”, Proceedings of the IEEE journal of solid-state circuit, (2004) May 4.

[11] R. Behzad, "Design of Analog CMOS Integrated Circuits”, McGraw Hill Higher Education, New York, (2000).

[12] W. Sansen and W. M. C, “Analog Design Essentials”, Editor, Springer-Verlag New York Inc, New York, (2006).

[13] S. S. Tan, L. K. Yeh and Y. H. Chiu, "An Integrated Low-Noise Sensing Circuit with Efficient Bias Stabilization for CMOS MEMS Capacitive Accelerometers”, Proceedings of the IEEE Transactions of Circuits and Systems, (2011) September 29.

[14] S. Hsu and M. Ker, "Dependence of Device Structures on Latch-up Immunity in a High Voltage 40V CMOS Process with Drain Extended MOSFET", Proceedings of the IEEE Transactions of Circuits and Systems, (2007) April 2. 